\title{
Modelo de negócios: constructo real ou metáfora de estratégia?
}

\author{
Luiz Antonio Joia* \\ Sinval Ferreira ${ }^{*}$
}

\begin{abstract}
Resumo
Este artigo analisa a discussão sobre os termos "modelo de negócios" e "estratégia", e seu principal objetivo é verificar se esses conceitos são distintos ou não. A maioria dos estudos até agora realizados defendem a tese de que sejam diferentes. 0 objetivo deste artigo é testar a veracidade dessa hipótese. Para isso, são comparadas abordagens consolidadas de estratégia, como a da escola de posicionamento e a visão baseada em recursos (resource-based view$\mathrm{RBV})$, com as definições de vários autores para o termo "modelo de negócios". Usando-se análise interpretativa de viés hermenêutico, conclui-se que os constructos não são totalmente diferentes, i.e., que existe sobreposição e nível de similaridade entre Estratégia e Modelo de Negócio. Da mesma forma, verifica-se que modelo de negócios é um instrumento dinâmico e sistêmico para várias abordagens de estratégia. Isso possibilita entrelaçá-las numa única definição, aumentando o potencial dessas abordagens para explicar e orientar o processo estratégico real das organizações.
\end{abstract}

Palavras-chave: modelo de negócios; estratégia; visão baseada em recursos; posicionamento estratégico; tecnologia da informação; negócios digitais.

\begin{abstract}
This article analyzes the discussion about the terms "business model" and "strategy", and aims mainly to verify whether these concepts are similar ones. Most of the studies already undertaken consider that business model and strategy are different constructs. Hence, the aim of this article is to test this hypothesis using an interpretative method based on hermeneutics. In order to accomplish this objective several definitions of business model are compared with two main strategic approaches, namely the Positioning school and the Resource Based View school. It is concluded that Business Model and Strategy are not entirely different constructs, i.e. there is some overlapping and similarity level between them. Furthermore, it is verified that business model acts as a dynamic and systemic tool integrating different strategic approaches and enabling different strategic concepts to be intertwined into a single definition, which increases the potential of these schools to explain and guide the actual strategic process within the organizations.
\end{abstract}

Keywords: business model; strategy; resource-based view; strategic positioning; information technology; digital business.

\section{Introdução}

A expressão modelo de negócios é cada vez mais usada tanto por acadêmicos como practitioners. O termo começou a ser utilizado com freqüência por consultores, executivos e acadêmicos, principalmente, depois que surgiram os negócios baseados na Internet (OSTERWALDER e PIGNEUR, 2002), transformando a expressão numa das mais populares no mundo dos negócios. Mesmo assim, modelo de negócios não possui uma definição clara e inequívoca, o que dificulta a compreensão de seu significado e tem limitado os estudos sobre o termo.

\footnotetext{
* Doutor em Ciências em Engenharia de Produção (Major: Inovação Tecnológica e Organização Industrial) pela COPPE/UFRJ. Professor da EBAPE/FGV. E-mail: luizjoia@ fgv.br. Endereço: Praia de Botafogo, 190 - sala 526 - Botafogo - Rio de Janeiro - RJ - 22250-900.

* Mestrando em Gestão Empresarial pela EBAPE/FGV. E- mail: sinvalferreira@ yahoo.com.br. Endereço: Pça. João Pinheiro 129/301 - Centro - Muriaé - MG Cep.:
} 36.880-000.

Artigo recebido em julho de 2005 e aceito para publicação em agosto de 2005. 
Como foi dito anteriormente, muitas definições sobre esse conceito vem surgindo. Acadêmicos como Rappa (2004), Hamel (2000) e Amit e Zott (2001), apenas para citar alguns, têm se empenhado em definir esse constructo, para adequar seu uso à prática empresarial. Essas várias definições têm em comum o fato de estarem muito próximas dos conceitos e modelos aplicados pelas teorias e instrumentos da área de estratégia. Isso tem levado a uma série de suposições sobre a semelhança, ou mesmo, sobre a sobreposição que existe entre os dois termos (SEDDON et al, 2004; MANSFIELD e FOURIE, 2004). Para Slywotzky (1996, p.4), por exemplo, trata-se da "maneira como uma companhia seleciona seus clientes, define e diferencia seus produtos e/ou serviços - de quais tarefas deverá se incumbir e quais irá terceirizar -, configura seus recursos, vai ao mercado, oferece novos produtos e/ou serviços e consegue ter lucro". Contudo, é difícil afirmar se tal assertiva refere-se a um modelo de negócios ou a uma estratégia.

Muitos são os autores que estudam a relação entre esses dois constructos (SEDDON et al, 2004; MANSFIELD e FOURIE, 2004; CHESBROUGH e ROSENBLOOM, 2002; ETHIRAJ, GULER e SINGH, 2000). Poucos, entretanto, admitem que os dois termos se sobrepõem, pois, na realidade acreditam que modelo de negócios seja uma metáfora de estratégia (HEDMAN e KALLING, 2003). Quase todos os autores envolvidos nessa discussão têm definido modelo de negócios como um novo constructo nos campos da administração e da tecnologia da informação. Dentre esses autores, podemos destacar Seddon (2004), Chesbrough e Rosenbloom (2002) e Magretta (2002). No entanto, até agora, seus estudos não se mostraram confiáveis, devido não apenas às diversas interpretações que as suas definições podem ter, quanto por problemas de validade do constructo.

Este artigo pretende contribuir para essa discussão, analisando se realmente é correto afirmar que modelo de negócios e estratégia são constructos diferentes. Assim, analisa vários conceitos atribuídos a modelo de negócios, verificando até que ponto diferem ou não daqueles relacionados à estratégia. Trata-se de uma análise importante, não apenas para os estudos sobre estratégia, mas também para o entendimento do que sejam sistemas e tecnologia da informação. Nesse sentido, a hipótese a ser testada considera que modelo de negócios seja um constructo diferente do de estratégia. Para essa checagem, é necessário responder às seguintes questões:

- modelo de negócios é um constructo totalmente diferente dos usados para definir estratégia?

- modelo de negócios insere novo conteúdo ou agrega valor às diversas abordagens sobre estratégia?

Assim, a questão levantada neste artigo pode ser colocada nos seguintes termos: qual a relação entre os constructos modelo de negócios e estratégia ? Para investigar essa questão, duas abordagens sobre estratégia foram selecionadas, determinando o perfil da pesquisa. A primeira é a da escola de posicionamento e foi escolhida por sua inequívoca importância acadêmica e empírica. A segunda, a da visão baseada em recursos (RBV), foi escolhida por sua crescente inserção em pesquisas relacionadas a sistemas e tecnologia da informação (KEARNS e LEDERER, 2003; BHARADWAJ, 2000), área que disseminou o uso do termo modelo de negócios (TIMMERS, 1988; ETHIRAJ, GULER e SINGH, 2000).

\section{Fundamentação teórica}

Para este estudo, é necessário ter uma visão de algumas das diversas abordagens sobre estratégia e discutir o conceito de modelo de negócios.

\section{Abordagens de estratégia}

O conceito de estratégia é um dos temas mais estudados por acadêmicos da administração, desde que esse campo de conhecimento tomou forma e ganhou relevância, tanto na academia, quanto no meio empresarial (GHEMAWAT, 2002; WRIGHT, KROLL e PARNELL, 2000). Como resultado, vários autores como Ansoff (1977), Porter (1986) e Barney (1991), dentre outros, desenvolveram suas próprias teorias sobre o conceito; algumas das quais marcaram definitivamente as decisões e os estudos relacionados às organizações (GHEMAWAT, 2002). 
A escola de posicionamento e a visão baseada em recursos são duas das várias abordagens sobre estratégia surgidas a partir da década de 1950. Aqui, elas serão descritas sinteticamente, sem que se pretenda esgotar o assunto; até porque não é objetivo deste artigo apresentar as limitações nem as críticas que vem sendo apontadas nessas abordagens.

\section{Abordagem de posicionamento}

Surgiu nos anos 1970, a partir de estudos do Boston Consulting Group (BCG) (GHEMAWAT, 2002). Possui modelos analíticos muito utilizados nas corporações, tais como: as cinco forças competitivas, a matriz BCG, as estratégias genéricas e a cadeia de valores. A premissa básica dessa escola é definir a estratégia a partir da análise do ambiente externo à organização (MINTZBERG, AHLSTRAND e LAMPEL, 2000).

O primeiro modelo desenvolvido pela escola de posicionamento foi a matriz crescimento/participação, conhecida como matriz BCG, numa alusão à entidade que a desenvolveu. De acordo com Schewe e Hiam (2000), esse instrumento tem como objetivo encontrar o portfólio ideal de produtos e serviços de uma organização, os quais são classificados segundo sua participação no mercado (market-share) e a taxa de crescimento da indústria a qual pertencem. Os produtos e serviços são, assim, divididos em quatro categorias: "estrela", "vaca leiteira", "interrogação" e "cachorro" (SCHEWE e HIAM, 2000; CHURCHIL JR. e PETER, 2003). De acordo com Ghemawat (2002), o objetivo da análise da matriz BCG é manter um equilíbrio entre as categorias "estrela", "vaca leiteira" e "interrogação", para que seja garantida a competitividade da organização, eliminando os produtos/serviços da categoria "cachorro" do portfólio da empresa.

No entanto, o maior destaque dessa escola são os conceitos desenvolvidos por Michael Porter, lançados principalmente através de seus livros (PORTER, 1986 e 1989). Porter (1996 e 1999) define estratégia como algo deliberado, que cria uma posição exclusiva e valiosa, envolvendo um diferente conjunto de atividades. Na visão desse autor (1991), a estratégia é obtida a partir de uma análise da atratividade do setor industrial onde a empresa opera - conseguida pela análise das cinco forças competitivas - e da atratividade da posição relativa da empresa. Tal atratividade é conseguida através da estratégia genérica, que estabelece a opção estratégica básica e a posição a ser ocupada no mercado, para se conseguir a vantagem competitiva. Porter (1991) afirma, ainda, que a organização deve configurar suas atividades e processos de forma distintiva, através da sua cadeia de valores, para ocupar a posição pretendida no mercado.

No modelo das cinco forças competitivas de Porter (1986), o ambiente competitivo da empresa é avaliado a partir dos fornecedores, dos novos entrantes, dos produtos substitutos, dos compradores e da concorrência. Com base na análise das cinco forças, Porter (1989) afirma que as empresas têm como opção duas estratégias genéricas: a da liderança em custo e a da diferenciação, havendo ainda uma terceira alternativa estratégica, um hibridismo das duas anteriores: a estratégia do foco.

Porter (1989) também lança o modelo da cadeia de valores, que classifica as atividades da organização em primárias (logística interna e externa, operações, marketing e vendas, e serviço), e de apoio e suporte (infraestrutura da empresa, gerência de recursos humanos, desenvolvimento de tecnologia e suprimentos). Esse modelo procura configurar as atividades organizacionais de forma original, credenciando a empresa a ocupar uma posição de valor no mercado.

Para Porter (1996, 1999 e 2001) existem seis princípios fundamentais para o posicionamento estratégico:

- a empresa deve começar com o objetivo certo: retorno superior sobre o investimento a longo prazo;

- a estratégia tem que habilitar a empresa a entregar uma proposta de valor diferente da apresentada pela concorrência;

- a estratégia tem que ser reflexo de uma distinta cadeia de valores;

- a estratégia tem que envolver trade-offs; ou seja, exercer opções, simultaneamente, de grande importância e excludentes, para se tornar única;

- a estratégia tem que definir como todos os elementos da organização irão se ajustar de forma conjunta; 
- a estratégia tem que envolver continuidade na direção escolhida.

Visão baseada em recursos (resource- based view - RBV)

Em 1959, com Penrose, surge a idéia da organização como um amplo conjunto de recursos (PENROSE, 1959; WERNERFELT, 1984). Ao contrário do que se viu na abordagem de posicionamento, o foco da RBV está num conjunto de recursos intra-organizacionais que podem gerar e sustentar vantagem competitiva, garantindo lucratividade por longos períodos. Segundo Teece, Pisano e Shuen (1997), a abordagem baseada em recursos específicos da firma preenche uma lacuna deixada pela abordagem de posicionamento, que subvaloriza o potencial dos recursos específicos da firma. Na abordagem de posicionamento, os recursos associados à criação de valor podem ser comprados pela organização. Já a RBV fundamenta-se na premissa de que as empresas são heterogêneas, e de que dispor desses recursos é uma vantagem de cada organização, pois a criação e o desenvolvimento de um recurso estão enraizados na sua própria complexidade. Tal fato, até certo ponto, inibe ou dificulta a mobilidade de recursos estratégicos.

Para Wernerfelt (1984), os recursos de uma firma podem ser tangíveis ou intangíveis, como capacidade de produção, liderança em custo, marcas, tecnologia, conhecimento tecnológico, mão-de-obra especializada, recursos financeiros, contatos comerciais e competência em processos, entre outros. Segundo Teece, Pisano e Shuen (1997), uma forma útil de identificar os principais recursos da empresa é através de uma análise de suas forças e fraquezas.

Apesar da evidente oposição entre o ponto de vista da escola de posicionamento e o da de recursos, alguns estudos recorrem ao modelo das cinco forças competitivas de Porter (1986 e 1999) para avaliar os recursos quanto à sua capacidade de permitir às empresas criarem barreiras de entrada, reduzirem custos de transação e serem atrativas num ambiente competitivo (WERNERFELT,1984).

Para Barney (2001), o valor de um recurso também é determinado pela sua interação com o ambiente competitivo da firma, o qual determina sua raridade e, portanto, sua capacidade de gerar e manter vantagem competitiva. Isso significa que permite avaliar se o recurso é específico ou não de uma determinada organização, e se é ou não uma competência distinta. Esse mesmo conceito de valor para uma determinada competência é compartilhado por outros estudos (TEECE e PISANO; 1994).

Segundo Barney (1995), para proporcionar e manter vantagem competitiva, um recurso deve ter alguns atributos, os quais foram definidos por um modelo, o VRIO. O nome é um acrônimo formado pelas iniciais de cada característica inerente aos recursos com potencial para gerar ou manter vantagem competitiva: "valioso" ter capacidade para gerar valor, "raro" - ser escasso no mercado, "inimitável" - ser difícil de ser replicado, imitado ou substituído adequadamente pelos concorrentes, e "organizável" - por poder ser organizado de forma que permita sua exploração. (ver figura 1).

\section{Figura 1}

\section{As características dos recursos no modelo VRIO}

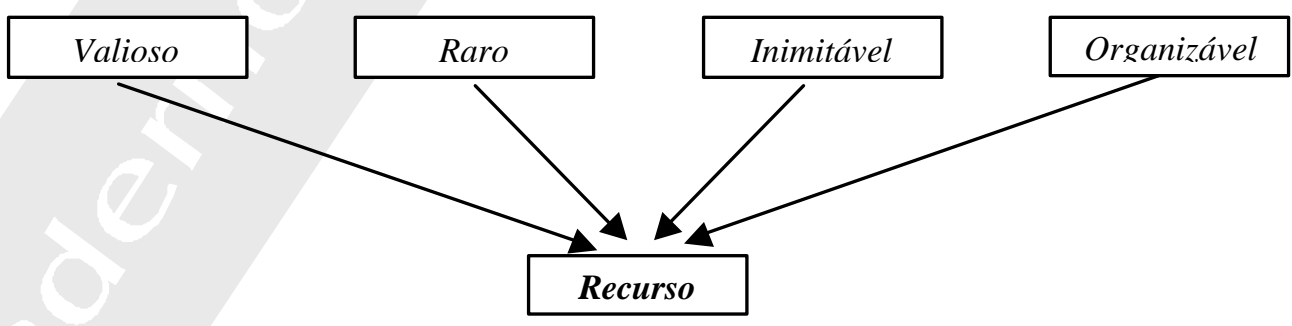

Fonte: elaborado pelos autores a partir de Barney (2001). 
Além disso, a RBV enfatiza a contínua acumulação (estocagem) de recursos como base para a vantagem competitiva. Essa lógica é estudada por Grant (1991), que descreveu graficamente esse processo como mostra a figura 2 .

\section{Figura 2}

\section{Modelo analítico de Grant}

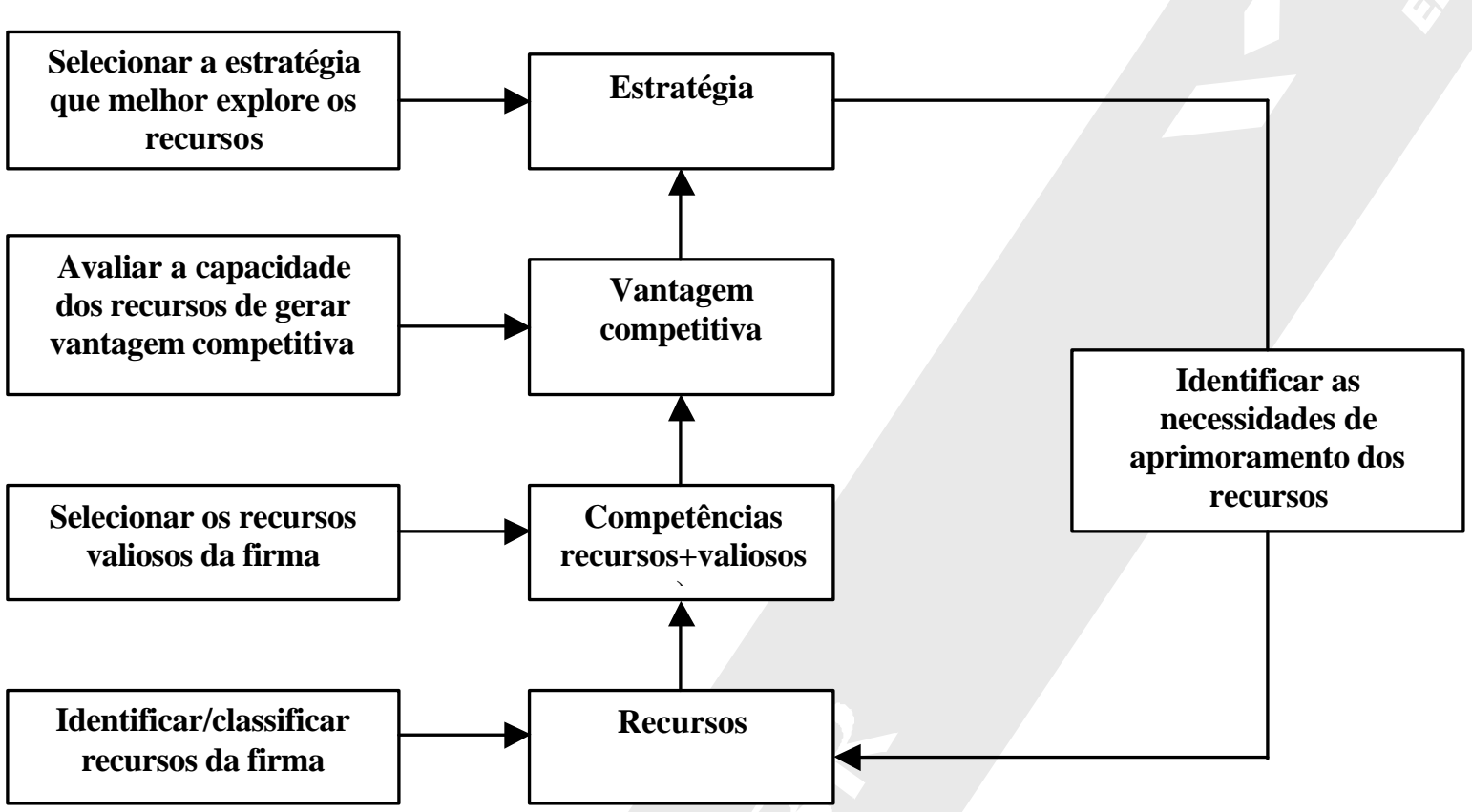

Fonte: adaptado de Grant (1991).

O modelo de Grant (1991) consiste, antes de tudo, na identificação dos recursos, seguida da respectiva classificação quanto à sua natureza. Na etapa seguinte, são avaliados os recursos, para que se selecione os mais valiosos, os quais são chamados por Grant (1991) de competências. No estágio posterior, busca-se identificar os recursos com maior capacidade de gerar e manter vantagem competitiva. Cumprida essa fase, a empresa seleciona a estratégia que possibilite uma exploração mais eficiente do potencial de seus recursos. Por fim, existe um processo de retroalimentação, onde a organização identifica a necessidade de aumentar a especialização dos recursos-chave de sua estratégia.

A excessiva preocupação com a acumulação ou criação de verdadeiros "estoques" de recursos levou à caracterização dessa abordagem como estática (BARNEY, 2001). Isso fez surgir uma visão dinâmica para a abordagem da RBV, chamada de "competências dinâmicas" (TEECE e PISANO, 1994; HELFAT e PETERAF, 2003).

Segundo Teece e Pisano (1994), o termo "competência" tem a finalidade de enfatizar o papel-chave do gerenciamento estratégico na função de adaptar, integrar e reconfigurar especialidades da empresa. $\mathrm{O}$ termo "dinâmicas" imputa o sentido de que as competências devem acompanhar e predizer as mudanças em seu ambiente, observando a velocidade das inovações e capacitando as empresas a responder, com velocidade, às demandas perceptíveis e veladas desse ambiente, criando inclusive novas competências (TEECE e PISANO, 1994; IANSITI e CLARK, 1994). 
Para Leonard-Barton (1998), as competências são responsáveis pela distinção entre empresas e pelo estabelecimento da vantagem competitiva. Iansiti e Clark (1994) dizem ainda que as competências dinâmicas são responsáveis pela sustentabilidade e pelo prolongamento dos períodos de performance positiva da empresa.

A acumulação, manutenção e geração de novas competências são condicionadas ao processo cumulativo de aprendizagem nas organizações (IANSITI e CLARK, 1994; KIM, 1998; FIGUEIREDO, 2003). Esse processo tem natureza incremental; ou seja, gera inovações de forma contínua e evolutiva, o que levou Tushman e O’Reilly III (1996 e 1999) a questionarem os estudos da abordagem de competências dinâmicas. Para esses autores, os ciclos tecnológicos são marcados por mudanças contínuas e descontínuas. Nesse sentido, as organizações estariam preparadas apenas para mudanças contínuas, não para as descontínuas, o que poderia ameaçar sua performance.

Alguns modelos analíticos têm sido desenvolvidos para explicar a criação de competências. O modelo de Leonard-Barton (1998) inclui como atividades geradoras de conhecimento a solução compartilhada de problemas, a implantação e integração de inovações e conhecimentos, a experimentação, a prototipagem e a importação de conhecimento de fontes externas. Teece e Pisano (1994) enfatizam os processos internos da firma, sua posição tecnológica e a trajetória de acumulação de conhecimento.

Iansiti e Clark (1994), por sua vez, destacam a integração da firma com as bases de conhecimento e os sinais do ambiente externo, e também sua própria integração interna. Segundo esses autores, a sinergia derivada da integração desse recursos pode propiciar a concepção de novos produtos, a geração de conhecimento e a implementação de idéias.

Por outro lado, a interação também está presente na abordagem de Pavitt (1990), o qual além de enfatizar a sinergia entre recursos como promotores de inovação, afirma que a implementação de novas idéias deve ser estimulada e que a capacidade de inovação de uma organização a credencia a continuar inovando no futuro, ou seja, criando e mantendo competências.

\section{Modelo de negócios}

Este tem sido um dos conceitos mais utilizados na literatura de gestão empresarial nos últimos anos. A popularidade do termo é comprovada quando, ao se pesquisar o termo no sistema de buscas Google (www.google.com), são encontradas nada menos do que cerca de 6.820 .000 ocorrências. Numa base de dados com características acadêmicas, como o Proquest (www.il.proquest.com/proquest/), foram encontrados 12.410 documentos associados ao termo em questão (em junho de 2005).

Apesar da popularidade do termo, pouco se sabe sobre sua origem. Entretanto, no início do século passado, Schumpeter (1934 e 1950) começou a fazer pesquisas no campo empresarial sobre a influência da combinação de fatores de produção - até então vistos como isolados e sem relação entre si - na geração de novos mercados, produtos e mesmo de setores industriais. Os fatores considerados nessas pesquisas e a própria abordagem dada pelo pesquisador têm relacionamento com os atuais componentes e definições de modelo de negócios. Assim, esses estudos podem ser considerados pioneiros em relação ao que viria mais tarde a ser denominado modelo de negócios.

Posteriormente, Gardner (1960), num estudo relativo à educação e formação de executivos, aborda o termo dando-lhe o sentido de incorporar todas as características de um determinado negócio. Em 1962, Raymond A. Hoffman, em seu livro Inventories: a guide to their control, costing, and effect upon income and taxes (apud EDWARDS, 1964), aborda a avaliação de inventários em vários modelos de negócios. Nesse livro, o termo assume o significado de "tipo de negócio", sendo empregado para identificar os vários tipos de negócio existentes naquele momento. Depois, Stanford (1972) retomou o uso do termo modelo de negócios, ligando-o à modelagem de ambientes de negócios em computador (simulação). Nesse sentido, modelo de negócios era tido como uma representação da realidade de um determinado negócio, ideal para análises de cenários e treinamentos de executivos. 
Na década de 1990, o termo modelo de negócios passa a receber variadas conceituações, sobretudo, a partir do momento em que a tecnologia da informação e, principalmente, a Internet passam a exercer forte influência na práxis negocial vigente (PATELI, 2002).

A pluralidade de definições para modelo de negócios aumenta a dificuldade para se chegar a um conceito único, impedindo que modelos teóricos consistentes sejam desenvolvidos. Dessa forma, o uso indiscriminado de diferentes concepções gera uma dissonância cognitiva em relação ao verdadeiro significado do constructo. Mesmo assim, Hedman e Kalling (2003) crêem que, apesar da indefinição, o termo é útil para explicar as relações entre gestão da informação, negócios eletrônicos e estratégia empresarial, áreas onde a popularidade do termo modelo de negócios cresce continuamente (HEDMAN e KALLING, 2003).

\section{Modelo de negócios versus estratégia}

Freqüentemente, os conceitos de modelo de negócios têm sido usados como sinônimos de estratégia (SEDDON et al, 2004; MANSFIELD e FOURIE, 2004; MAGRETTA, 2002). A maior parte das definições genéricas de modelo de negócios são muito próximas das principais definições e abordagens de estratégia, o que será visto adiante. Isso tem gerado dúvidas e debates sobre se são constructos diferentes ou não.

Seddon (2004) e Seddon e Lewis (2003), por exemplo, afirmam que modelo de negócios é apenas uma abstração de estratégia, mas consideram apenas a visão de estratégia da escola de posicionamento. Ademais, a análise desses autores sofre influência da conclusão a que querem chegar, já que ignoram instrumentos importantes da própria escola de posicionamento (por exemplo, a cadeia de valores), além de outras relevantes abordagens de estratégia, como a escola da visão baseada em recursos.

Outros autores, como Magretta (2002), embora definam modelo de negócios literalmente como estratégia, chegaram à conclusão de que são conceitos diferentes. De acordo com Magretta (2002), esses constructos diferem porque, ao contrário de modelo de negócios, estratégia considera a dimensão da competição. Contudo, o argumento da autora é passível de crítica, pois entender modelo de negócios como um sistema tem por objetivo definir como as peças/recursos de um negócio devem ser reunidos; isto é, está condicionada a uma prévia avaliação dos mesmos, da forma como podem ser combinados e com que finalidade. Esses recursos, segundo Grant (1991), são avaliados com base em análises interempresas, ou seja, com base no ambiente de negócios. Além disso, reunir recursos ou peças tem que ser feito com um objetivo, que pode ser criar ou manter uma vantagem competitiva, obter maior eficiência dentro do segmento de negócio em que se atua, ou mesmo gerar mais valor para os clientes da organização. Todas essas finalidades, ainda que discretamente, dizem respeito a uma análise que envolve a dimensão da competição.

Portanto, como pode ser percebido, os estudos a esse respeito enfrentam uma série de dúvidas quanto a sua validade interna. Para enriquecer o debate acerca do grau de superposição existente entre os dois conceitos, este artigo procura verificar se esses constructos podem ser considerados distintos ou não. Para tanto, algumas definições de modelo de negócios foram selecionadas e são apresentadas adiante para uma análise interpretativa de viés hermenêutico, confrontada com duas abordagens consagradas de pensamento estratégico. Dessa forma, a hipótese nula de pesquisa a ser testada, gerada a partir do referencial teórico apresentado é:

H0: estratégia e modelo de negócios são constructos totalmente distintos entre si.

\section{Método de pesquisa}

Este artigo é resultado de uma revisão de literatura que abrange duas abordagens sobre estratégia e vários conceitos associados a modelo de negócios. A pesquisa recorre à análise interpretativa de viés hermenêutico para verificar se modelo de negócios e estratégia são constructos totalmente diferentes entre si. 
Num primeiro momento, foram levantados trabalhos acadêmicos que de alguma forma definem modelo de negócios. A partir desse levantamento, foram selecionadas algumas definições para serem analisadas. Os critérios para a escolha foram os seguintes:

- conceitos originários de pesquisas relevantes, publicadas em periódicos reconhecidos pela significativa contribuição à geração de conhecimento na área;

- conceitos de fácil entendimento tanto para acadêmicos quanto para practitioners; $\mathrm{e}$

- conceitos que não apenas definem modelo de negócios, mas que também especificam seus componentes.

Paralelamente a essa fase, foi revista a bibliografia sobre uma das mais importantes escolas de estratégia, a de posicionamento. Logo após, foi feita a revisão da escola RBV, por ser objeto de inúmeras pesquisas recentes (WERNERFELT, 1995; CONNER e PRAHALAD, 1996; HELFAT e PETERAF, 2003), o que a torna um importante arcabouço teórico, utilizado tanto por acadêmicos quanto por empresas e consultorias. Além disso, a visão baseada em recurso também se destaca como a abordagem a que cada vez mais se recorre nas pesquisas sobre tecnologia da informação (KEARNS e LEDERER, 2003; BHARADWAJ, 2000), que está diretamente relacionada ao surgimento do termo modelo de negócios (TIMMERS, 1988; ETHIRAJ, GULER e SINGH, 2000).

Depois de formulada a base teórica, as definições de modelo de negócios escolhidas foram comparadas (por análise interpretativa) com o corpo teórico de cada uma das duas abordagens de estratégia.

De acordo com Klein e Myers (1999, p.69), uma análise pode ser caracterizada como interpretativa se assume que o caminho para se chegar ao conhecimento da realidade é através de construções sociais como a linguagem, a consciência, o entendimento compartilhado, a interpretação de textos, de documentos, ferramentas e de outros artefatos. Nessa análise, não há variáveis dependentes ou independentes, mas sim uma preocupação com o sentido que o ser humano dá às situações e conceitos quando eles emergem (KAPLAN e MAXWELL, 1994).

O interpretativismo incorpora vários métodos de pesquisa (Klein e Myers, 1999), sendo a hermenêutica o método de pesquisa adotado neste estudo. Como a hermenêutica é a arte ou ciência da interpretação, e como o foco desta pesquisa é a interpretação e comparação de textos dentro de contextos preestabelecidos, a adoção desse método se justifica e se mostra bastante adequada (OLSON e CARLISLE, 2001).

Portanto, feita a seleção das definições de modelo de negócios (segundo os critérios apresentados anteriormente) e das principais características de duas das principais abordagens de estratégia, é aplicado o círculo hermenêutico (GADAMER, 1976). Esse círculo procura decompor em partes o texto selecionado, de modo que essas partes sejam interpretadas, seguindo-se a essa interpretação uma visão sistêmica do conjunto, para que em seguida, seja feita uma nova decomposição, num moto contínuo de decomposição/sistematização (GADAMER, 1976). Assim, trechos de textos maiores abordando o conceito de modelo de negócios são escolhidos, interpretados e comparados com as características das escolas estratégicas selecionadas, para que se tenha uma visão do conjunto. A partir daí, esse conjunto é novamente decomposto para que seja verificado o quanto essa interpretação é adequada ou não.

Para interpretar e confrontar os conceitos de modelo de negócios com as escolas de estratégia escolhidas é seguido o arcabouço teórico proposto por Vries e Miller (1987), que visa tornar aplicável o método hermenêutico. Esse arcabouço é composto das seguintes regras, conforme mostra a tabela 1: 
Tabela 1

Regras de Vries e Miller para interpretação de textos

\begin{tabular}{ll}
\hline Unidade temática & $\begin{array}{l}\text { Observações coletadas e modeladas de forma inter- } \\
\text { relacional, em busca de comunalidades e de uma } \\
\text { unidade coesa }\end{array}$ \\
\hline Reconhecimento de padrões & $\begin{array}{l}\text { Busca de estruturas paralelas, entre as definições de um } \\
\text { constructo e entre constructos, que levem à emergência } \\
\text { de padrões temáticos }\end{array}$ \\
\hline Urgência psicológica & $\begin{array}{l}\text { Busca e identificação, nos textos analisados, das } \\
\text { situações que demandaram maior atenção e pressão dos } \\
\text { autores desses textos }\end{array}$ \\
\hline Funções múltiplas & $\begin{array}{l}\text { Análise dos possíveis significados que o texto possa } \\
\text { ter, interpretando-o a partir de múltiplas perspectivas }\end{array}$
\end{tabular}

Fonte: Vries e Miller (1987); Olson e Carlisle (2001).

Portanto, os textos conceituando modelo de negócios foram analisados para se chegar à unidade temática "estratégia". Em seguida, procurou-se reconhecer padrões de linguagem relacionados com as abordagens estratégicas usadas. O objetivo era retirar dos textos, partes que revelavam urgência psicológica dos respectivos autores, associadas ao constructo estratégia. Posteriormente, as múltiplas perspectivas das definições sobre modelo de negócios escolhidas foram analisadas e confrontadas com as escolas estratégicas adotadas. Foi solicitado a colegas acadêmicos que as interpretassem e, então, feita a comparação entre essas interpretações e a dos autores deste artigo, com o intuito de se evitar a tendenciosidade.

Segundo Walsham (1995), um dos grandes problemas da análise interpretativa é estar presente num mundo científico dominado pelo positivismo, o que é corroborado por, entre outros, Orlikowski e Baroudi (1991) e Iivari (1991). Dessa forma, recorrer à retórica para conduzir a hermenêutica de textos pode ser um método pouco consistente diante dos estudos de viés nomotético que entendem que todos os fenômenos naturais têm relações de causalidade passíveis de serem descobertas - sem dubiedade e dependência dos intérpretes -, a partir de dados "objetivos" recolhidos pelo pesquisador (WALSHAM,1995, p.376).

No entanto, o próprio Walsham (1995, p.378) acredita que o interpretativismo pode ser um melhor método para estudar as áreas onde impera a tecnologia e os sistemas de informação (como em modelo de negócios) do que os métodos positivistas. Por outro lado, o método aplicado não deve ser confundido com deconstrucionismo (DERRIDA,1976), porque muitas vezes, parece estar levantando ceticismo, decepção ou interesses/motivações específicos por trás de uma definição específica.

Finalmente, a escolha arbitrária pelos autores de sete definições de modelo de negócios e de duas escolas estratégicas deve ser considerada como uma limitação do método adotado.

\section{Análise interpretativa}

Adiante, são apresentadas algumas definições de modelo de negócios com as respectivas análises interpretativas confrontadas com as abordagens conceituais sobre estratégia, de acordo com o método de pesquisa proposto anteriormente.

\section{Abordagem de Lumpkin \& Dess (2004)}

Numa abordagem de viés tecnológico, esses autores definem modelo de negócios na Internet como um método e um conjunto de ações que explicam como um negócio cria valor e gera lucros num ambiente competitivo, tendo como principal objetivo propiciar e garantir vantagem competitiva à empresa que o adotar. 
Quando Lumpkin e Dess afirmam que um modelo de negócios explica como a firma cria valor e gera lucros em seu ambiente, diretamente estão se valendo da abordagem estratégica de posicionamento, pois o ponto de referência dessa abordagem, tanto para valor como para lucro, é a comparação da firma com seu ambiente (PORTER, 1986). Para Lumpkin e Dess, os modelos de negócios têm características que são únicas, mesmo em ambientes digitais.

Quando se afirma que alguns modelos de negócios podem ter características únicas, novamente se percebe a presença da escola estratégica de posicionamento, já que a diferenciação (uma das estratégias genéricas) tem justamente esse objetivo (PORTER, 1986, p.51). Essa definição também está relacionada com a visão baseada em recursos, pois características únicas estão diretamente associadas com o valor dos recursos de que a organização dispõe. Esses recursos, por sua vez, ainda podem ser combinados para que gerem uma proposição de valor distinta (LEONARD-BARTON, 1998).

\section{Abordagem de Rappa (2004)}

Para esse autor, um modelo de negócios é um método de fazer negócios. Ele afirma que todo modelo de negócios "especifica o que uma companhia faz para criar valor, como está situada na cadeia de valores de seu segmento, como se relaciona com seus parceiros nessa cadeia e o tipo de arranjo que tem com seus clientes para gerar receita" (p.34).

A citação que Rappa faz da cadeia de valores aponta claramente para a visão intra-organizacional da escola de posicionamento. Sua definição de modelo de negócios é muito próxima (para não dizer idêntica) da que Porter (1989) faz do sistema de valores, onde são analisados tanto a posição da empresa na cadeia, quanto o relacionamento desta com as cadeias de valores dos parceiros que estão à sua frente e/ou atrás no sistema produtivo. Porter (1989) também prevê o arranjo das atividades identificadas na cadeia de valores visando atingir o cliente, para resultar em mais receita e valor para a empresa.

Nesse artigo, Rappa (2004), revela que em determinadas indústrias, os métodos para se fazer negócios podem variar, mas que existem limites impostos por fatores tecnológicos, pela dinâmica competitiva entre empresas (e entre empresas e seus parceiros) e pelas expectativas e preferências de seus clientes. Essa última observação está relacionada com o modelo das cinco forças de Porter (1986), já que demonstra as restrições que um modelo de negócios pode sofrer, causadas por um ambiente competitivo.

\section{Abordagem de Hedman \& Kalling (2003)}

Esses autores propõem um modelo de negócios genérico, formado pelos seguintes componentes: clientes, competidores, portfólio de produtos/serviços para a venda, atividades e sua organização, recursos, fornecedores e escopo de gerenciamento. Vários desses componentes estão presentes praticamente em todas as abordagens de estratégia apresentadas neste artigo.

O componente "recursos" é inteiramente identificado com a visão baseada em recursos. Hedman e Kalling chegam a estabelecer essa relação, considerando ainda os "fornecedores" como uma possível fonte de recursos valiosos. As "atividades" são componentes relacionados à cadeia de valores, o que também é reconhecido pelos dois autores. O componente "atividades e sua organização", ainda de acordo com Hedman e Kalling, é idêntico ao proposto por Porter (1989) na escola de posicionamento. Já "portfólio de produtos/serviços para venda" é condicionado e determinado pela definição da estratégia genérica de Porter (1989) - o que também é observado por Hedman e Kalling (2003) -, havendo assim uma relação entre esse componente e a matriz BCG da escola de posicionamento.

\section{Abordagem de Chaharbaghi, Fendt e Willis (2003)}

Na visão desses autores, modelo de negócios é uma "representação do pensamento gerencial e de práticas que ajudam a se ter uma visão de negócio, bem como no entendimento e na execução de atividades, de forma distinta e específica" (p.373). Ao observar que as atividades devem ser executadas de forma distinta e específica, eles fazem uma conexão direta com a cadeia de valores, já que Porter (1989, p.102), na escola de 
posicionamento, propõe exatamente isso; ou seja, uma configuração das atividades que ofereça uma proposta de valor diferenciada.

Chaharbaghi, Fendt e Willis consideram os modelos de negócio ferramentas vitais para representar as características essenciais da realidade, tanto para gerar idéias, quanto para desempenhar um importante papel no processo decisório. Para os autores, todos os modelos de negócio são contextualizados nos ambientes em que operam, sendo validados nessa ambiência. Dessa perspectiva, é o contexto (o ambiente competitivo) que dá significado e determina a validade e a utilidade de um modelo de negócios. Essa enfoque tem relação direta com o modelo das cinco forças de Porter (1989), onde o ambiente determina o negócio e a estratégia a ser seguida. Nesse sentido, modelo de negócios é a estratégia originada da análise ambiental, foco da escola de posicionamento.

\section{Abordagem de Betz (2002)}

Esse autor começa seu artigo (p.21) dizendo que "um modelo de negócios é uma abstração de um negócio lucrativo, é sua maneira de gerar dinheiro". Essa definição vai ao encontro da definição de estratégia (SEDDON et al, 2004) segundo a qual modelos de negócio são abstrações de um negócio. Por sua vez, uma abstração é a representação de algumas partes de um todo; isto é, uma visão parcial. Como modelo de negócios identifica a configuração necessária para gerar lucro (BETZ, 2002), infere-se que ele é a própria configuração das atividades da organização que permitem conseguir essa lucratividade, identificando-se com o modelo da cadeia de valores da escola de posicionamento.

Por outro lado, a forma de obter lucro pode estar atrelada a algum recurso valioso, raro, inimitável e organizável da organização, o que direciona o conceito para a visão baseada em recursos (BARNEY, 1995).

Para Betz, um modelo de negócios é "uma lista sistemática de políticas que guiarão as futuras especificações de inputs, outputs, processos e o valor da operação completa dos negócios da corporação" (p.21). Na visão desse autor, qualquer modelo de negócios pode ser gerado a partir da combinação distinta de quatro fatores: recursos, vendas, lucro e capital.

Nessa nova passagem de Betz, percebe-se claramente modelo de negócios como uma configuração de recursos que, combinados, podem gerar competências distintas para a organização, o que identifica o conceito com a abordagem da visão baseada em recursos.

Betz coloca modelo de negócios no mesmo nível de importância e utilidade de conhecidos instrumentos da escola de posicionamento, como a cadeia de valores de Porter (1989) e a matriz BCG. Betz vai ainda mais longe ao afirmar que tais instrumentos são modelos de negócio. Como exemplo, declara que "o modelo de Porter é apenas um dos tipos de modelo de negócios que podem ser utilizados em planejamento estratégico" (p.21).

Para Betz, como pode ser claramente interpretado, modelo de negócios nada mais é do que mais um dos muitos componentes que formam o constructo estratégia.

\section{Abordagem de Chesbrough \& Rosenbloom (2002)}

Nesse estudo, os autores afirmam que um modelo de negócios provê um arcabouço coerente que abrange características tecnológicas e potenciais inputs, convertendo-os (através dos clientes e dos mercados) em resultados econômicos. Além disso, tem como finalidade focalizar inovações situadas entre o desenvolvimento tecnológico e a criação de valor econômico. Em outro trecho do artigo (p.529), Chesbrough e Rosenbloom dizem que

Um modelo de negócios de sucesso cria uma lógica heurística que conecta o potencial técnico com a realização de valor econômico. Os modelos de negócio revelam o valor latente de uma tecnologia, mas essa lógica restringe a subseqüente busca por novos e alternativos modelos para explorar outras tecnologias mais recentes. 
As definições apresentadas mostram que a percepção de seus autores quanto ao que seja modelo de negócios está ligada à abordagem da visão baseada em recursos. $\mathrm{O}$ foco na inovação mostra isso claramente, pois a abordagem das competências dinâmicas - ligada à visão baseada em recursos - tem direcionado os estudos estratégicos para a prendizagem e a inovação nas organizações (LEONARD-BARTON, 1998; TEECE e PISANO, 1994; IANSITI e CLARK, 1994; PAVITT, 1990; KIM, 1998; FIGUEIREDO, 2003). Ao mesmo tempo, a definição de modelo de negócios estabelecida por Chesbrough e Rosenbloom revela incoerências, pois se num dado momento definem o conceito como heurístico, em outro, o consideram como limitador da inovação. Chesbrough e Rosenbloom dizem que estratégia é diferente de modelos de negócio em, pelo menos, três pontos:

- os modelos de negócios têm seu foco direcionado para a criação de valor para o cliente e para a entrega desse valor. A estratégia, por outro lado, enfatiza a sustentabilidade desse valor, considerando as possíveis ameaças, por exemplo, de novos entrantes e/ou produtos substitutos;

- um modelo de negócios não abrange a dimensão financeira da geração de valor para o acionista. Já a estratégia foca justamente essa dimensão financeira; e

- os modelos de negócios assumem conscientemente que o conhecimento organizacional é cognitivamente limitado, sendo corroído pelo sucesso anterior da organização; o que não acontece com a estratégia.

Quanto a essas diferenças, alguns equívocos merecem ser destacados. No primeira assertiva, a existência de proposta de valor para o consumidor pressupõe a existência desse valor em relação ao que está disponível no mercado onde a organização atua. Além disso, a sustentabilidade associada a um modelo de negócios está implícita no fato dessa proposta de valor ser contínua. Para que isso aconteça, é necessário um constante monitoramento do mercado e a renovação da proposta de valor, para que haja adequação às novas condições ambientais.

A segunda assertiva é de fácil contestação, haja vista que nenhum dos modelos associados às diversas abordagens de estratégia relacionadas neste artigo apresentam especificamente uma dimensão financeira. Ao mesmo tempo, todos os modelos destacam a relevância desse ponto. $\mathrm{O}$ mesmo acontece com os modelos de negócios. Não se pode afirmar que um modelo de negócios despreze a performance financeira de uma empresa por não considerá-la seu componente explícito. A dimensão financeira embasa a análise de qualquer modelo de negócios, estratégia ou ação de uma empresa, pois é nela que se verifica a viabilidade econômica de um empreendimento (COPELAND, KOLLER e MURRIN, 2002).

A terceira assertiva pode ser contestada por Tushman e O'Reilly III (1996), quando descrevem a síndrome do sucesso. Atribuída à estratégia, ela diz respeito exatamente à limitação cognitiva do aprendizado organizacional, a qual é atribuída por Chesbrough e Rosenbloom (2002) aos modelos de negócios. Assim, este artigo refuta completamente a diferença entre estratégia e modelo de negócios.

\section{Abordagem de Amit e Zott (2001)}

$\mathrm{Na}$ interpretação desses autores, modelo de negócios é uma configuração estrutural dos componentes de uma transação, desenhada para explorar as oportunidades de negócios e válida tanto para empresas que atuam em mercados virtuais, quanto em mercados convencionais. Através dessa concepção, Amit e Zott prescrevem os modelos de negócio para qualquer tipo de empresa. Ademais, sua definição resulta de uma combinação dinâmica dos modelos que Porter (1991) prescreve para estratégia, onde é analisado o ambiente (modelo das cinco forças), definida qual a melhor estratégia (entre as genéricas) para explorar as oportunidades e, por fim, é promovida uma configuração das atividades que melhor se ajustam à estratégia (cadeia de valores). Portanto, é uma definição que segue, fielmente, a escola estratégica de posicionamento. 


\section{Figura 3}

\section{Componentes de um modelo de negócios aplicado ao comércio eletrônico}

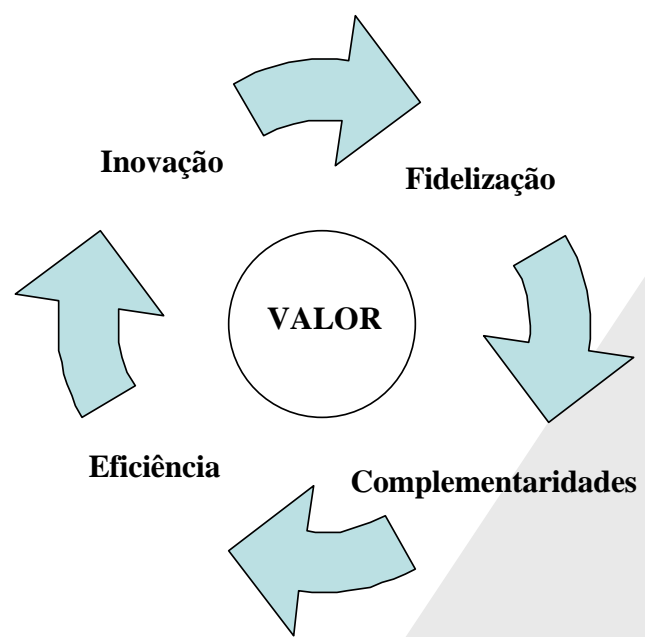

Fonte: adaptado de Amit e Zott (2001).

O modelo de Amit e Zott, apresentado na figura 3, tem como objetivo gerar modelos de negócios de alto valor agregado. Seus componentes - entre os quais, a fidelização do cliente - também são encontrados nos modelos de estratégia, a cujas várias escolas estão relacionados.

O conceito de valor, que possibilita ao modelo ser valioso no mercado, tem a mesma base conceitual que o de recurso valioso de Barney (1995), da visão baseada em recursos. Além disso, a forma como os componentes são organizados e a nomenclatura empregada por Amit e Zott em seu modelo seguem os mesmos princípios da escola de posicionamento, através da cadeia de valores de Porter (1989). Nesta, as atividades primárias, juntamente com as de apoio, são configuradas de forma adequada para gerar uma proposta diferenciada de valor. Dessa forma, esse modelo, que inicialmente parecia não se enquadrar no conceito de estratégia, se mostra fiel tanto à visão baseada em recursos, quanto à abordagem estratégica de posicionamento.

\section{Resultados consolidados e conclusões}

A comparação do conceito de modelo de negócios não com uma, mas com duas abordagens sobre o pensamento estratégico, leva a algumas conclusões quanto à indagação inicialmente levantada neste artigo; ou seja: modelo de negócios e estratégia são ou não são constructos distintos?

De acordo com a tabela 2, fica claro que não são termos completamente diferentes, uma vez que todas as definições de modelo de negócios relacionadas foram identificadas com uma ou com as duas abordagens de estratégia. Por isso, é insustentável a hipótese nula $(\mathrm{HN})$ de que ambos sejam constructos totalmente distintos. 
Tabela 2

Consolidação da análise "modelo de negócios versus estratégia"

\begin{tabular}{|c|c|c|}
\hline \multirow{2}{*}{$\begin{array}{l}\text { DEFINIÇÕES DE MODELOS } \\
\text { DE NEGÓCIOS }\end{array}$} & \multicolumn{2}{|c|}{ ABORDAGENS DE ESTRATÉGIAS } \\
\hline & Posicionamento & Visão baseada em recursos \\
\hline Lumpkin e Dess & $\mathrm{X}$ & $\mathrm{X}$ \\
\hline Rappa & $\mathrm{X}$ & \\
\hline Hedman e Kalling & $\mathrm{X}$ & $\mathrm{X}$ \\
\hline Chaharbaghi, Fendt e Willis & $\mathrm{X}$ & 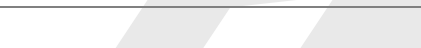 \\
\hline Betz & $\mathrm{X}$ & $\mathrm{X}$ \\
\hline Chesbrough e Rosembloom & & $\mathrm{X}$ \\
\hline Amit e Zott & $\mathrm{X}$ & $\mathrm{X}$ \\
\hline
\end{tabular}

Fonte: Autores.

A análise consolidada permite ainda outras conclusões sobre a relação entre os dois termos. A primeira referese a estudos anteriores com a mesma finalidade deste artigo (SEDDON et al, 2004; MAGRETTA, 2002). Fica claro que uma única abordagem de estratégia não consegue abranger o conceito de modelo de negócios, por causa, antes de tudo, da própria natureza dessa discussão. Como Mintzberg e Quinn (1996) afirmam, não existe uma única definição para estratégia, e isso leva a uma disputa teórica em torno do tema que tem provocado o surgimento de várias abordagens distintas. Mintzberg, Ahlstrand e Lampel (2000), por exemplo, descrevem nada menos do que 10 abordagens diferentes, o que dificulta comparações.

Do mesmo modo que em relação a estratégia, é possível concluir que é muito variável a definição do constructo modelo de negócios. Como o termo é muito recente na literatura especializada - pois ganhou destaque somente com o advento da economia digital (TIMMERS, 1988; ETHIRAJ, GULER e SINGH, 2000) -, o fato de vários autores procurarem construir sua própria definição também dificulta comparações. Nesse sentido, tal diversidade de conceitos corrobora os argumentos contrários à idéia de que modelo de negócios e estratégia são totalmente diferentes. Da mesma forma que existem modelos de negócios genéricos, específicos, compostos etc., também existem estratégias genéricas, específicas, funcionais, de curto e longo prazo, entre outras. Isso revela uma grande variação quanto à amplitude, temporalidade e grau de abrangência, tanto em termos de modelo de negócios quanto de estratégia. Quando não se compara esses dois termos no mesmo nível de análise, corre-se o risco de erros de interpretação que levam a conclusões equivocadas, como as das análises de Chesbrough e Rosenbloom (2002), Seddon (2004) e Magretta (2002).

Já que uma única visão sobre estratégia é insuficiente para ser confrontada com modelo de negócios, é possível inferir que este se refere a uma visão sistêmica e dinâmica das abordagens de estratégia existentes. Podemos afirmar ainda que numa única definição de modelo de negócios encontra-se uma ou todas as abordagens de estratégia apresentadas neste estudo. Por conseguinte, a utilidade dessa combinação está tanto em aumentar o poder explicativo das teorias de estratégia, quanto em permitir uma aplicação mais fácil destas, através de uma visão mais próxima da realidade e dos aspectos importantes de um determinado negócio.

Dessa forma, o emprego de um modelo de negócios potencializa os pontos fortes de cada escola de estratégia, minimizando seus pontos fracos através da complementaridade entre elas. Como os conceitos de modelo de negócios são flexíveis e fáceis de aplicar, acabam possibilitando que várias abordagens de estratégia sejam empregadas simultaneamente, uma vez que estejam reunidas num único conceito, o que aproxima ainda mais a teoria de estratégia do dia-a-dia das organizações. A conclusão a que se chega é a de que modelo de negócios é um instrumento dinâmico e sistêmico de estratégia, altamente adaptável à realidade das empresas.

Mais uma vez, é fundamental ressaltar que em todos os momentos da análise interpretativa percebe-se que os conceitos de estratégia e de modelo de negócios se sobrepõem. Em nenhum momento modelo de negócios apresentou contribuições teóricas que estratégia não contemplasse. Os modelos de negócios utilizaram definições de cada escola de estratégia, em maior ou menor intensidade, conforme a tendência teórica dos 
autores, ou de sua finalidade. Quaisquer que sejam as definições de modelo de negócios (por mais diferentes que sejam), elas estão sempre alinhadas com alguma definição de estratégia. Por isso não são constructos completamente distintos.

Do exposto anteriormente, é possível afirmar que modelo de negócios agrega valor às várias abordagens sobre estratégia; não de forma individualizada, mas de maneira dinâmica e sistêmica, permitindo promover uma interação entre as várias escolas de pensamento estratégico existentes.

\section{Estudos futuros}

Uma vez comprovado que os constructos não são totalmente diferentes, é importante que futuras pesquisas procurem medir o grau de similaridade entre esses conceitos, avaliando até que ponto estão sobrepostos.

De uma perspectiva analítica, pode-se relacionar as seguintes proposições relativas a essa questão, também representadas na figura 4:

- P1: estratégia e modelo de negócios são constructos totalmente distintos;

- P2: estratégia e modelo de negócios não são constructos totalmente distintos;

- P2a: estratégia e modelo de negócios são constructos que, embora apresentem uma interseção, mantém algumas diferenças entre si;

- P2b: estratégia e modelo de negócios são constructos totalmente idênticos;

- P2c: modelo de negócios é um constructo inteiramente contido no constructo estratégia;

- P2d: estratégia é um constructo inteiramente contido no constructo modelo de negócios.

Figura 4

Proposições lógicas entre os constructos "estratégia” e "modelo de negócios"

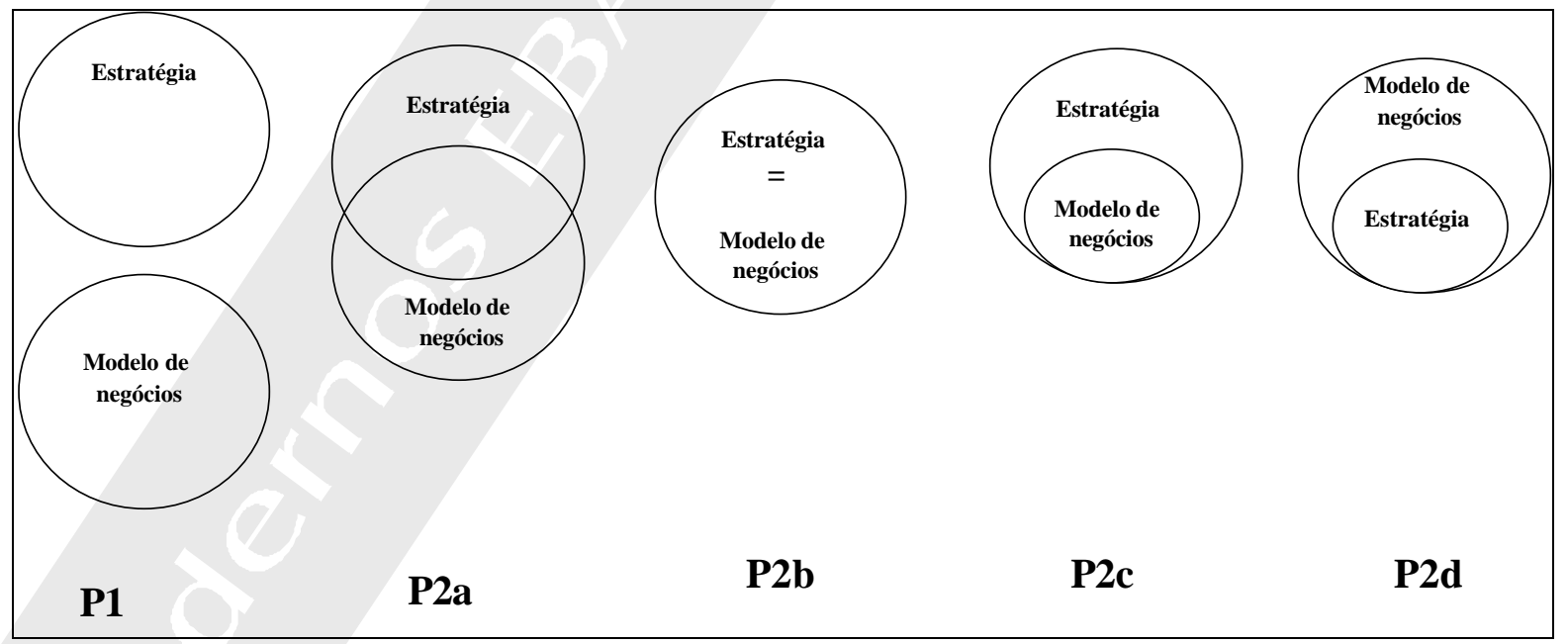

Fonte: Autores. 
Este estudo discorda da proposição 1 (“estratégia e modelo de negócios são constructos totalmente distintos”) $\mathrm{P} 1:\{\mathrm{E}\} \cap\{\mathrm{MN}\}=\{\varnothing\}$. Entretanto, as seguintes proposições derivadas de P2, em linguagem lógica da teoria dos conjuntos, ficaram em aberto, e devem ser analisadas em futuros estudos:

P2a: $[[\{\mathrm{E}\} \cap\{\mathrm{MN}\} \neq\{\varnothing\}] \wedge[(\sim \mathrm{P} 2 \mathrm{~b}) \mathrm{V}(\sim \mathrm{P} 2 \mathrm{c}) \mathrm{V}(\sim \mathrm{P} 2 \mathrm{~d})]]$

P2b: $\{\mathrm{E}\}=\{\mathrm{MN}\}$

P2c: $\{\mathrm{E}\} \supset\{\mathrm{MN}\}$

P2d: $\{\mathrm{E}\} \subset\{\mathrm{MN}\}$

Onde:

$\{\mathrm{E}\}=$ estratégia

$\{\mathrm{MN}\}=$ modelo de negócios

Para consolidar os resultados deste estudo e como contribuição à evolução da linha de pesquisa aqui adotada, sugerimos que futuros estudos abordem temas como o uso de mais definições de modelo de negócios e outras abordagens sobre o pensamento estratégico.

Finalmente, cabe observar que é bem-vindo todo estudo empírico que vise a uma efetiva compreensão por parte dos executivos sobre o que seja modelo de negócios e que compare diferentes conceitos sobre o tema com as definições mencionadas neste artigo. 


\section{Referências bibliográficas}

AMIT, R.; ZOTT, C. Value creation in e-business. Strategic Management Journal, v.22, p.493-520, 2001.

ANSOFF, H. I. Estratégia empresarial. São Paulo: McGraw-Hill do Brasil,1977.

BARNEY, J. B. Firm resources and sustained competitive advantage. Journal of Management, v.17, n.1, 1991. . Looking inside for competitive advantage. The Academy of Management Executive, v.9, n.4, Nov., 1995.

Is the resource-based "view" a useful perspective for strategic management research? Yes. The Academy of Management Review, v.26, n.1, 2001.

BETZ, F. Strategic business models. Engineering Management Journal, v.14, n.1, 2002.

BHARADWAJ, A. S. A resource-based perspective on information technology capability and firm performance: an empirical investigation. MIS Quarterly, v.24, n.1, p.169-196, Mar. 2000.

CHAHARBAGHI, K.; FENDT, C.; WILLIS, R. Meaning, legitimacy and impact of business models in fast-moving environments. Management Decision, v.41, n.4, 2003.

CHESBROUGH, H.; ROSENBLOOM, R. S. The role of the business model in capturing value from innovation: evidence from Xerox Corporation's technology spin-off companies. Industrial and Corporate Change, v.11, n.3, 2002.

CHURCHILL JR., G. A.; PETER, J. P. Marketing: criando valor para o cliente. São Paulo: Saraiva, 2003.

CONNER, K. R.; PRAHALAD, C. K. A resource- based theory of the firm: Knowledge versus opportunism. Organization Science, v.7, n.5, 1996.

COPELAND, T.; KOLLER, T.; MURRIN, J. Avaliação de empresas - valuation: calculando e gerenciando o valor das empresas. 3. ed. São Paulo: Makron Books, 2002.

DERRIDA, J. De la grammatologie. [S.I.]: Les Editions de Minuit, 1967.

EDWARDS, J. D. Review 13 - no title. The Journal of Business, v.37, n.1, 1964.

ETHIRAJ, S.; GULER, I.; SINGH, H. The impact of Internet and electronic technologies on firms and its implications for competitive advantage. The Wharton School. Research paper, 2000. Disponível em: <http://commerce.concordia.ca/gkersten/ec_papers/models/ 00Ethiraj_models.pdf >. Acesso em: 15 maio 2004.

FIGUEIREDO, P. N. Learning, capability accumulation and firms differences: evidence from latecomer steel. Industrial and Corporate Change, v.12, n.3, 2003.

GADAMER, H. G. Philosophical hermeneutics. Berkeley: University of California Press, 1976.

GARDNER, J. M. Educators, electrons and business models: a problem in synthesis. Accounting Review, v.35, n.4, 1960.

GHEM AWAT, P. Competition and business strategy in historical perspective. Business History Review, v.76, p.37-74, spring, 2002.

GRANT, R. M. The resource-based theory of competitive advantage. California Management Review, v.33, n.3, 1991.

HAMEL, G. Liderando a revolução. Rio de Janeiro: Campus, 2000.

HEDMAN, J.; KALLING, T. The business model concept: theoretical underpinnings and empirical illustrations. European Journal of Information Systems, v.12, p.49-59, 2003.

HELFAT, C. E.; PETERAF, M. A. The dynamic resource-based view: capability lifecycles. Strategic Management Journal, v.24, n.10, 2003.

IANSITI, M.; CLARK, K. B. Integration and dynamic capability: evidence from product development in automobiles and mainframe computers. Industrial and Corporate Change, v.33, n.3, 1994.

IIVARI, J., A paradigmatic analysis of contemporary schools of IS development. European Journal of Information Systems, v.1, n.4, 1991.

KAPLAN, B.; MAXWEL, J. A. Qualitative research methods for evaluating computer information systems. In: ANDERSON, J. G.; AYDIN, C. E.; JAY, S. J. (Ed.). Evaluating health care information systems: methods and applications. Thousand Oaks: Sage, p.45-68, 1994.

KEARNS, G. S.; LEDERER, A. L. A resource based view of strategic IT alignment: how knowledge sharing creates competitive advantage. Decision Science, v.34, n.1, 2003.

KIM, L. Crisis construction and organizational learning: capability building in catching-up at Hyundai Motor. Organization Science, v.9, n.7, 1998.

KLEIN, H. K.; MYERS, M. D. A set of principles for conducting and evaluating interpretive field studies in information systems. MIS Quarterly, v.23, p.67-96, 1999.

LEONARD-BARTON, D. Nascentes do saber: criando e sustentando as fontes de inovação. Rio de Janeiro: Fundação Getulio Vargas, 1998. 
LUM PKIN, G. T.; DESS, G. G. E-business strategies and Internet business models: how internet adds value. Organizational Dynamics, v.33, n.2, p.161-173, 2004.

MAGRETTA, J. Why business models matter. Harvard Business Review, v.80, n.5, 2002.

MANSFIELD, G. M.; FOURIE, L. C. H. Strategy and business models - strange bedfellows? A case for convergence and its evolution into strategic architecture. South African Journal of Business Management, v.35, n.1, p.35-44, March 2004.

MINTZBERG, H.; AHLSTRAND, B.; LAM PEL, J. Safári de estratégia: um roteiro pela selva do planejamento estratégico. Porto Alegre: Bookman, 2000.

; QUINN, J. B. The strategy process- concepts, contexts, cases. 3. ed. New Jersey: Prentice Hall, 1996.

OLSON, D.; CARLISLE, J. Hermeneutics in information systems. Americas Conference on Information Systems, 2001.

ORLIKOWSKI W.; BAROUDI J. J. Studying information technology in organizations: research approaches and assumptions. Information Systems Research, v.2, n.1, p.1-28, 1991.

OSTERWALDER, A.; PIGNEUR, Y. An e-business model ontology for modeling e-business. In. BLED ELECTRONIC COMM ERCE CONFERENCE, 15., 2002, Bled, Slovenia, 17-19 J une 2002.

PATELI, A. A domain area report on business models. Athens University of Economics and Business, 2002. (White paper, version 1.3).

PAVITT, K. What we know about the strategic management of technology. California M anagement Review, v.32, n.2, 1990.

PENROSE, E. T. The theory of the growth of the firm. New York: John Wiley $\&$ Sons, 1959.

PORTER, M. E. Estratégia competitiva: técnicas para análise de indústrias e da concorrência. 17. ed. Rio de Janeiro: Campus, 1986.

. Vantagem competitiva: criando e sustentando um desempenho superior. 22. ed. Rio de Janeiro: Campus, 1989.

Towards a dynamic theory of strategy. Strategic Management Journal, v.12, [número especial], p.95-117, 1991.

What is strategy? Harvard Business Review, v.74, n.6, 1996.

Competição: on competition. Estratégias competitivas essenciais. 2. ed. Rio de Janeiro: Campus, 1999.

Strategy and the internet. Harvard Business Review, v.79, n.03, p.63-78, 2001.

RAPPA, M. A. The utility business model and the future of computing services. IBM Systems Journal, v.43, n.1, 2004.

SCHEWE, C. D., HIAM , A. MBA: curso prático marketing. Rio de Janeiro: Campus, 2000.

SCHUM PETER, J A. The theory of economic development. Cambridge: Harvard University Press, 1934.

Capitalism, socialism, and democracy. 3. ed. New York: Harper \& Row, 1950.

SEDDON, P. B. et al. The case for viewing business models as abstractions of strategy. Communications of the Association for Information Systems, v.13, Apr. 2004.

; LEWIS, G. P. Strategy and business models: what's the difference? PACIFIC ASIA CONFERENCE ON INFORMATION SYSTEMS, 7. $\overline{1} \overline{0}-\overline{1} \overline{3}$ July 2003.

SLYWOTZKY, A. J. Value migration: how to think several moves ahead of the competition. Boston, MA: Harvard Business School Press, 1996.

STANFORD, R. A. Ceteris Paribus methodology and computerized economics-business models. The Journal of Economic Education, v.4, n.1, Fall, 1972.

TEECE, D. J; PISANO, G. The dynamic capabilities of firms: an introduction. Industrial Corporate Change, v.3, n.3, 1994.

; SHUEN, A. Dynamic capabilities and strategic management. Strategic Management Journal. v. 18, n.7, p.509-533, 1997.

TIM MERS, P. Business models for electronic markets. Journal of Electronic Markets, v.8, n.2, p.3-8, 1988.

TUSHMAN, M. L.; O'REILLY III, C. A. Ambidextrous organizations: managing evolutionary and revolutionary change. California Management Review. v.38, n.4, 1996.

Building ambidextrous organizations. Health Forum Journal, v.42, n.2, 1999.

VRIES, M F. R. K.; MILLER, D. Interpreting organizational texts. Journal of Management Studies, v.24, n.3, 1987.

WALSHAM, G. The emergence of interpretivism in IS research. Information Systems Research, v.6, n.4, p.376-394, 1995.

WERNERFELT, B. A resource-based view of the firm. Strategic Management Journal, v.5, n.2, 1984.

A resource-based view of the firm: ten years after. Strategic Management Journal, v.16, n.3, 1995.

WRIGHT, P.; KROLL, M. J .; PARNELL, J. Administração estratégica: conceitos. São Paulo: Atlas, 2000. 